\title{
Comparative Analysis between Wavelets for the Identification of Pathological Voices
}

\author{
Náthalee Cavalcanti ${ }^{1}$, Sandro Silva ${ }^{1}$, Adriano Bresolin ${ }^{2}$, \\ Heliana Bezerra ${ }^{1}$, and Ana Maria G. Guerreiro ${ }^{1}$ \\ ${ }^{1}$ UFRN, Federal University of Rio grande do Norte, Brazil \\ nathalee.telecom@gmail.com, sandro@ct.ufrn.br, \\ heliana@ufrnet.br, anamaria@dca.ufrn.br \\ ${ }^{2}$ UTFPR, Federal Technological University of Paraná, Brazil \\ aabresolin@utfpr.edu.br
}

\begin{abstract}
This study presents a comparative analysis of wavelets, in order to find a descriptor that provides a better classification of voice pathologies. Different types of Wavelet Packet Transform were used as a tool for feature extraction and Support Vector Machine (SVM) to classify vocal disorders. Tests were conducted with 23 wavelets types in two SVMs, the first using the strategy "one vs. all" to classify normal and pathological voices and the second, using the strategy "one vs. one" to classify pathologies: edema and nodules. The best results were obtained using Daubechies family, especially Daubechies 5 (db5) wavelet.
\end{abstract}

Keywords: Vocal disorder, Wavelet Packet Transform, Support Vector Machine (SVM).

\section{Introduction}

Pathological voices have become a social concern, since the voice and speech are important in certain professions as teachers, speakers, singers, and quality of life in general. Moreover, the voice is an important tool of communication.

There is a great range of diseases that causes modifications in the voice. They could appear as a modification of the excitation morphology (the distribution of mass on vocal fold is increased). These are classified as organic pathologies as nodules, polyps, cysts and edemas. Voice disorders can also be caused by other pathologies which are provoked by neuro-degenerative diseases [1], [2].

The presence of pathologies in the vocal folds such as nodules, polyps, cysts and paralysis of laryngeal nerves can be corrected by: voice therapy, surgery, and in some cases, radiotherapy [3]. But not always vocal diseases cause necessarily changes in voice quality level perception of listeners.

Digital signal processing techniques have been used by acoustic analysis, as an effective noninvasive tool for diagnosing changes in sound production caused by diseases of the larynx, the voice classification of diseases and their pre-detection, aiding, this way, the development of the therapeutic process [4]. 
The aim of this paper is to present a comparative analysis of wavelets, used as input attributes of support vector machines, which will be responsible for classification of pathological voices. The vocal pathologies considered in this study were edemas and nodules.

Several studies on pattern recognition have used wavelet packet descriptors in the stage of feature extraction [5]-[7], but have not yet conducted studies on which the wavelets perform better in the classification of laryngeal pathologies.

The wavelet transform has been an important tool in signal and imaging processing to determine not just "which" frequency, but also "where" these frequencies are located [8].

The Wavelet Packet Transform has been used in recent years in signal processing allowing better classification of signs. This technique is used as a tool to extract features of signals from one or more dimensions improving the performance of the classifiers to extract relevant features through decomposition of signals in different frequency bands. In this work, we decide to use the Packet Wavelet Transform, which is a generalization of Discrete Wavelet Transform.

The SVM has aimed at building optimal hyperplanes which have the largest margin of separation between classes. The generated hyperplane is determined by a subset of points in classes, named support vectors. SVMs were used as a tool for classification of normal and pathological voices.

The main contribution of this paper is the application of these tools in the classification of pathological voices.

This paper is organized as follows: Section 2 presents the signal pre-processing phase. Section 3 shows the speech feature extraction through Wavelet Packet Transform. In Section 4, is the procedure of training and validation SVM. Section 5 shows the experiments and results in 10 tests with 23 types of wavelets.

\section{Signal Pre-processing}

The signal pre-processing is composed of several processes such as filtering, preemphasis, normalization and windowing. The aim of this step is to eliminate noise, discontinuities and any effects that might affect system performance.

The speech signals used in this work are a sustained vowel /a/. Initially, the voice signal is passed through a bandpass filter with a cutoff frequency of $80 \mathrm{~Hz}$ and $9 \mathrm{kHz}$, which will eliminate the signal frequencies above $9 \mathrm{kHz}$ and the noise of the electric grid $(60 \mathrm{~Hz})$. After that, the speech signal is pre-emphasized to equalize the spectrum of the speech signal and improve the spectral analysis performance [9]. The value of pre-emphasis filtering in time domain is 0.97 . In the normalization step, the speech signal is normalized by the maximum value of its amplitude.

Finally, the speech signal is broken into time frames at intervals of 16 to $32 \mathrm{~ms}$, so that the signal during this interval is considered stationary. Aiming to standardize and reduce the amount of processing and storage, the window width was set at $20 \mathrm{~ms}$, overlapping 33 percent. According to Stephen Levison [10], overlapping 25 percent is mathematically optimal, but experiments in this study the superposition of $33.3 \%$ had a better cost benefit due to reduced numbers of windows and the consequent reduction of processing time without significant loss of performance. Each frame is multiplied 
by Hamming window in order to minimize any signal discontinuities in the time domain, i.e to reduce abrupt transitions at the end of the signal.

\section{Wavelet Packet Transform}

The Wavelet Transform is used to analyze the non-stationary phenomena, such as voice. The bases of this transform are analyzed in the time domain and frequency domain and their algorithms process information at different scales, managed to get an image or a sign of a general and detailed way [11].

Through the wavelet can be seen that there are significant differences between normal voices and voices with nodules as shown in Fig. 1.

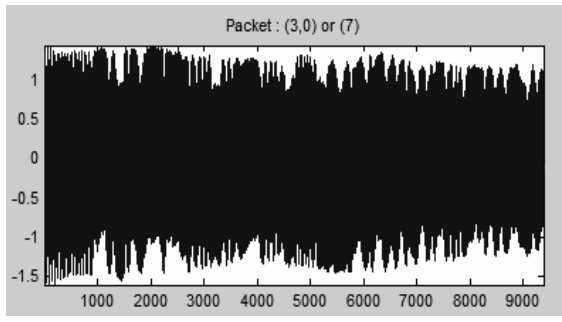

(a)

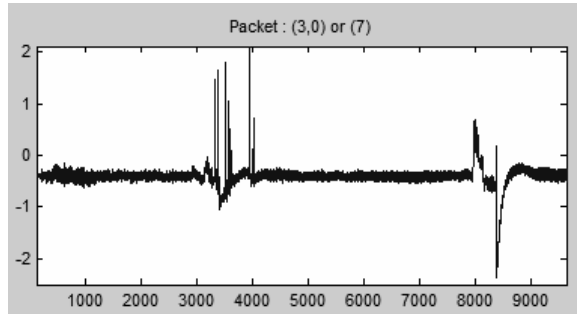

(b)

Fig. 1. (a) Signal of normal voice and (b) Voice Signal with Nodule

The Wavelet Transform Packet type used in this work is a generalization of the Discrete Wavelet Transform. Discrete Wavelet analysis on the signal is divided into approximation and detail coefficients, although only the approximation coefficients are divided again [12]. In both Wavelet Packet coefficients of approximation and the details are decomposed at each level as shown in Fig 2.

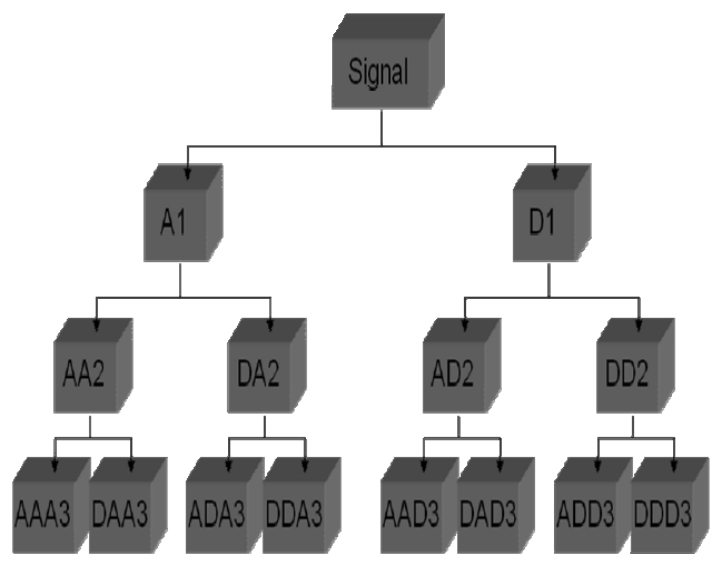

Fig. 2. Binary tree Wavelet Packet Transform with 3 levels of decomposition 


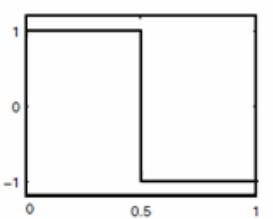

(a)

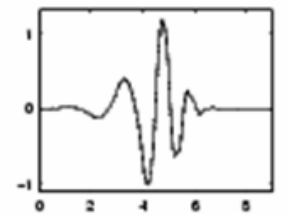

(b)

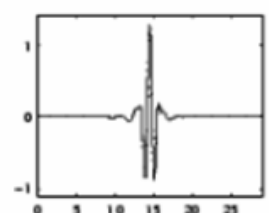

(c)

Fig. 3. Wavelets Mothers: (a) Haar; (b) Daubechies 5; (c) Coiflet 5

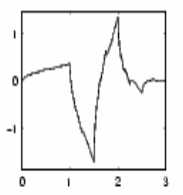

sym2

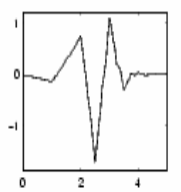

sym3

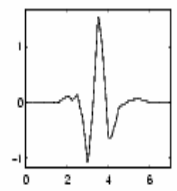

sym4

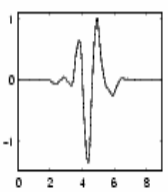

sym5

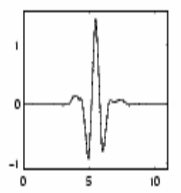

sym6

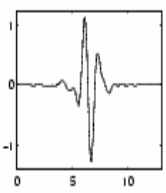

sym7

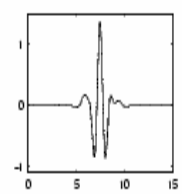

sym8

Fig. 4. Symlets Wavelets Examples

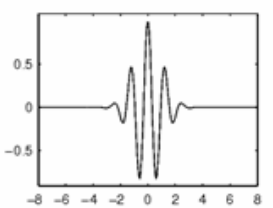

(a)

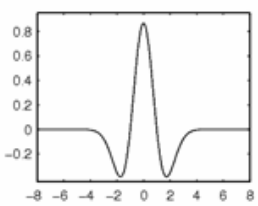

(b)

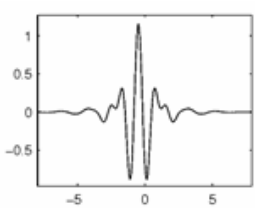

(c)

Fig. 5. (a) Morlet; (b) Mexican Hat; (c) Meyer

With this transform is possible to extract relevant characteristics of the signal, improving the classifiers performance by decomposing the speech signal into different frequency bands.

Depending on the characteristics of the signal to be analyzed, a different mother wavelet can be used. In Fig 3, 4 and 5 show kinds of wavelets used in the experiments. They are: Haar, Daubechies (db), Coiflets, Symlets, Morlet, Mexican Hat and Meyer.

\section{SVM Classifier}

Support Vector Machines (SVMs) is a technique based on statistical learning theory. This strategy was introduced by Vapnik in [13]. 
The SVM finds a single hyperplane with maximum margin of separation is denoted by $\delta$. This hyperplane lines located between $\mathrm{H} 1$ and $\mathrm{H} 2$, and will be great if the distance to the two lines is maximum. The H1 line defines the edge or border of objects of class +1 , while $\mathrm{H} 2$ line determines the edge or border with the objects of the class 1. Two objects Class +1 define the boundary line $\mathrm{H} 1$ and $\mathrm{H} 2$ to the border there are three objects of class -1 . These objects are called Support Vectors (Fig. 6).

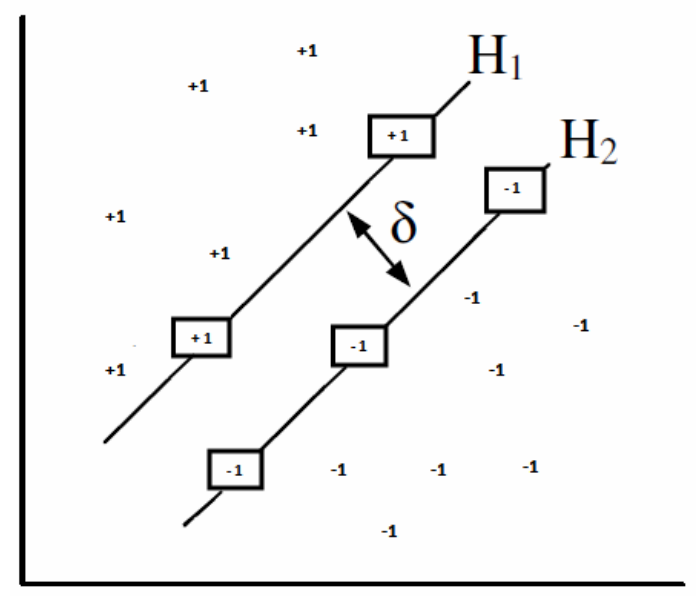

Fig. 6. Formation of the separation hyperplane through the support vectors

Basically, SVM maps the input space into a high dimensional space and from the calculation of an optimal separating hyperplane in this new space, the SVM learns the boundary between regions belonging to two classes. This separation hyperplane is chosen so that it maximizes the separation between the closest training samples [14].

Fig. 7. shows the SVMs used for pathological voices classification, where the first machine uses the strategy "one vs. all" (normal $x$ all) and the second machine is used if the first machine has classified certain voice to "All", i.e, unable to determine the type of pathology. This second machine uses the strategy "one vs. one" (edema $x$ nodule).

Each of the two machines can be considered as a binary classifier, they present as a result only 2 hypotheses: (+1) that corresponds to the first class and (-1) corresponding to the second class trained.

If the first machine (normal against all) presents the result +1 then the unidentified pattern is classified as normal. On the other hand, if the answer machine is -1 then the unknown pattern is classified as "all" or "not normal".

To identify pathologies is used machine 2 with strategy "one against one" that is "edema against nodule". The same as machine 1, if the SVM 2 presents +1 as result, the pathology is classified as edema, if the result is -1 , nodule is the correct classification of the pathologies. 


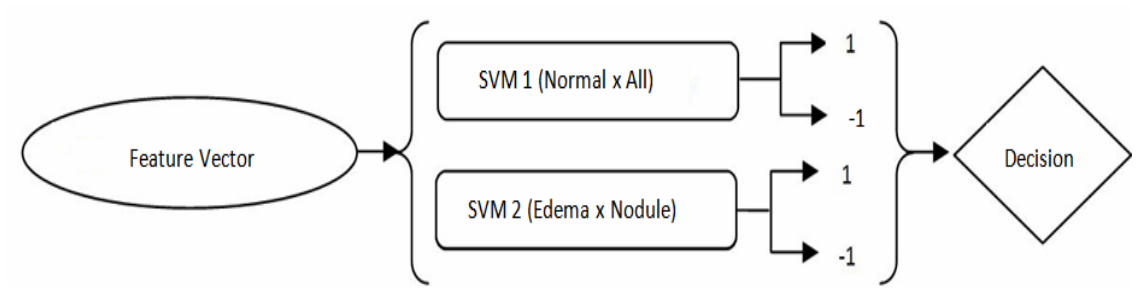

Fig. 7. SVM for three classes classification: Normal, Edema and Nodules

\section{Experiments and Results}

In this article are considered three classes: normal (normal voice, with no pathology in the vocal folds), edema (voices with vocal folds edema) and nodule (voices affected with nodules on vocal cords).

The database consists of 26 voices among which 10 with healthy voices, 10 presenting edemas in the vocal folds and 6 with nodules.

To form the feature vector were used coefficients obtained through the Wavelet Packet Transform. These data were used as input of SVMs. For each test performed, a new mother wavelet was tested to find that had better results.

Table 1. Performance Evaluation of two support vector machines

\begin{tabular}{ccc}
\hline \hline Wavelet type & $\begin{array}{c}\text { SVM 1 } \\
\text { \%SR mean }\end{array}$ & $\begin{array}{c}\text { SVM 2 } \\
\text { \%SR mean }\end{array}$ \\
\hline Daubechies 1 & 97,7 & 98,44 \\
Daubechies 2 & 96,62 & 98,32 \\
Daubechies 3 & 97,81 & 97,46 \\
Daubechies 4 & 98,46 & 98,66 \\
Daubechies 5 & 98,68 & 98,75 \\
Daubechies 6 & 98,14 & 97,68 \\
Daubechies 7 & 98,36 & 98,04 \\
Daubechies 8 & 97,89 & 98,46 \\
Coiflet 1 & 92,74 & 96,81 \\
Coiflet2 & 90,97 & 97,04 \\
Coiflet3 & 91,03 & 97,08 \\
Coiflet4 & 92,53 & 96 \\
Coiflet5 & 97,03 & 96,57 \\
Symlet 2 & 92,93 & 93,83 \\
Symlet 3 & 96,95 & 94,8 \\
Symlet 4 & 95,04 & 94,86 \\
Symlet 5 & 91,46 & 94,18 \\
Symlet 6 & 94 & 94,06 \\
Symlet 7 & 91,56 & 95,18 \\
Symlet 8 & 93,60 & 93,01 \\
Morlet & 93,81 & 92,48 \\
Mexican Hat & 92,57 & 85,99 \\
Meyer & 97,38 & 97,01 \\
\hline \hline
\end{tabular}


Two machines SVM 1 and SVM 2 were created. For each of these machines were carried out 10 tests with 23 different wavelets and their samples were chosen randomly, with 50 percent of samples for training and 50 percent for validation. This process is called cross-validation.

Table 1 shows the results when comparing normal and pathological voices (SVM 1) and pathological voices together (edema and nodules). Column 1 shows the mother wavelet used features descriptors. Columns 2 and 3 show the average performance of the device obtained after carrying out 10 tests. All results are expressed in success rate percentage $(\% \mathrm{SR})$.

The best results of classification for the two machines were obtained using wavelet Daubechies 5 with $98.68 \%$ in the first machine and $98.75 \%$ in the second machine. On machine 1, were obtained good results with the Daubechies wavelets $(4,7,6,8,3$ and 1) in ascending order of performance, followed by Meyer and Coiflet 5 . On the second machine, the other Daubechies wavelets (4, 8, 1 and 2), followed by Coiflet 5 Wavelets.

These results show that the Daubechies families are a good descriptor of voice pathologies classification, especially Daubechies 5. Moreover, it can be observed that the Daubechies 5 is also quite effective in speech recognition as in [15].

\section{Conclusions}

This paper analyzes different types of mother wavelets as feature descriptors in the classification of voice pathologies.

The main objective of this paper was to find the mother wavelet which best classifies normal and pathological voices. We performed extensive testing with voices from different patients with normal voice, edema and nodules. Through the mean shown in Table I can be seen that that the best results were obtained using Wavelet Daubechies Familie, especially Daubechies 5 for both machines. With other types of wavelets, we obtained good results with Meyer and Coiflet 5.

\section{Acknowlegment}

The authors thank to CAPES by support financed given.

\section{References}

1. Davis, S.B.: Acoustic Characteristics of Normal and Pathological Voices. Speech and Language: Advances in Basic Research and Practice 1, 271-335 (1979)

2. Quek, F., Harper, M., Haciahmetoglou, Y., Chen, L., Raming, L.O.: Speech pauses and gestural holds in Parkinson's disease. In: Proc. of International Conference on Spoken Language Processing, pp. 2485-2488 (2002)

3. Martinez, C.E., Rufiner, H.L.: Acoustic Analysis of Speech for Detection of Laryngeal Pathologies. In: Proceedings of the 22nd Annual International Conference of IEEE Engineering in Medicine and Biology Society, Chicago, EUA, vol. 3, pp. 2369-2372 (2000) 
4. Costa, S.C., Correia, S., Falcão, H., Almeida, N., Assis, F.: Uso da Entropia na Discriminação de Vozes Patológicas. In: II Congresso de Inovação da Rede Norte Nordeste de Educação Tecnológica, João Pessoa, Paraíba (2007)

5. Soares, H.B., Neto, A.D.D., de Carvalho, M.A.G.: Extration and Selection of Characteristics in Skin Lesions Images Using Wavelet in Multiresolution Analysis. In: ASME 2007 Summer Bioengineering Conference, SBC 2007 (2007)

6. Bresolin, A.A., Neto, A.D.D., Alsina, P.J.: Brasilian Vowels Recongnition using a New Hierarchical Decision Structure with Wavelet Packet and SVM. In: IEEE Intern. Conf. on Acoustics, Speech and Signal Processing. ICASSP 2007, vol. 2, pp. 493-496 (April 2007)

7. Kim, J., Cho, S., Choi, J.: Iris Recognition Using Wavelet Features. Journal of VLSI Signal Processing 38, 147-156 (2004)

8. de Almeida, N.C., Barros, J.D., Soares, H.B., Bresolin, A.A., Guerreiro, A.M.G., Brandão, G.B.: A new computational tool for voice analysis based on FFT, Wavelet Transform and Spectrogram. In: Proceedings of the ASME 2009 Summer Bioengineering Conference, SBC 2009 (2009)

9. Yang, S., Er, M.J., Gao, Y.: A high performance neural-networks-based speech recognition system. In: International Joint Conference on Neural Networks, IJCNN 2001, vol. 2, pp. 1527-1531 (July 2001)

10. Levison, S.C.: Mathematical Models for Speech Technology. John Wiley \& Sons Ltd., West Sussex (2005)

11. Júnior, A.J.P., Castilho, J.E.: Um estudo comparative entre a Análise de Fourier e Análise Wavelet. FAMAT em Revista - Número 05- Setembro (2005)

12. Parraga, A.: Aplicação da Transformada Wavelet Packet na Análise e Classificação de vozes patológicas. UFRGS, Porto Alegre (2002)

13. Vapnik, V.N.: Principles of risk minimization for learning thoery. In: Advances in Neural Information Processing Systems, San Mateo, CA, vol. 4, pp. 831-838 (1992)

14. Haykin, S.: Redes Neurais: Princípios e Prática. 2a Edição, Porto Alegre, Editora Bookman (2001)

15. Bresolin, A.A., Neto, A.D.D., Alsina, P.J.: Digit recognition using wavelet and SVM in Brazilian Portuguese. In: Proceedings of IEEE International Conference on Acoustics, Speech and Signal Processing, ICASSP 2008, Las Vegas, USA, pp. 1545-1548 (2008) 